\title{
PROJECTION VS. PERCEPTION : LA PRÉSENTATION DE SOI À TRAVERS LA DESCRIPTION DE L'AUTRE EN DIDACTIQUE DU FLE
}

\author{
MÉLANIE BUCHART \\ Université de Helsinki, Finlande
}

\begin{abstract}
Résumé : Dans cet article, nous proposons de problématiser les notions d'identité et d'altérité du point de vue de la didactique de l'interculturel en FLE et de l'analyse du discours. Après un rapide exposé des apports de ces autres disciplines concernant ces notions, nous nous concentrerons plus particulièrement sur les représentations de l'identité collective en classe de langue. En effet, à travers la mise en discours de l'altérité, l'énonciateur (par exemple l'auteur d'un manuel de FLE) diffuse des représentations figées à la fois sur l'autre, l'étranger, mais aussi sur la culture source c'est-à-dire sur sa communauté d'appartenance. Le chercheur peut ainsi déceler, à travers le discours des manuels locaux, ce que la société source remet en question, valorise ou déplore et, par conséquent, peut trouver intéressant, bizarre, 'anormal', dans la société cible. Le discours produit met en miroir deux mondes qui se rencontrent à travers un jeu constant de représentations endogènes et exogènes, dont nous montrerons quelques exemples tirés d'une analyse diachronique des manuels de FLE utilisés en Finlande depuis une cinquantaine d'années.
\end{abstract}

Mots-clés : identité, altérité, manuels, didactique, représentations

\section{INTRODUCTION}

Les notions d'identité et d'altérité ne peuvent se départir l'une de l'autre. Comme l'affirme Porcher (1997 : 21-22), 'l'autre est à la fois ma condition et ma définition', il n'existe pas de subjectivité sans intersubjectivité, pas de 'je' sans 'tu', pas d'ego sans alter ni d'alter sans ego et par conséquent pas d'identité sans altérité.

Dans cet article, nous proposons de problématiser ces notions d'identité et d'altérité du point de vue de la didactique de l'interculturel en FLE, et plus particulièrement des représentations de l'identité collective en classe de langue. En effet, à travers la mise en discours de l'altérité, l'énonciateur (par exemple l'auteur d'un manuel scolaire) diffuse des représentations figées à la fois sur l'autre, l'étranger, mais aussi sur la culture source qui met en discours ces représentations. Le discours produit met en miroir deux mondes qui se rencontrent à travers un 
jeu constant de représentations endogènes et exogènes et de rectifications de l'identité collective perçue par l'autre, dont nous montrerons quelques exemples tirés de manuels de FLE publiés en Finlande.

\section{L'IDENTITÉ : UNE PERCEPTION ?}

Le psychologue Erikson a mis en circulation et popularisé le terme d'identité' dans les sciences humaines. Ses premiers travaux remontent aux années 1930, suite à sa découverte de l'école 'culture et personnalité' (Kardiner, Mead, etc.). Dans les années 1950, en sociologie et en psychologie sociale, la théorie des rôles et la théorie du groupe de référence ont contribué à la diffusion de la notion d'identité, en insistant notamment sur le phénomène d'identification de l'individu à différents groupes (ethniques, religieux, politiques) (Halpern, 2004 : 14). Dans les années 1960, aux Etats-Unis, le terme s'est diffusé d'autant plus vite en sociologie que de multiples revendications identitaires l'ont définitivement installé dans le champ politique. L'identité est alors devenue un objet de recherches universitaires qui portent encore aujourd'hui principalement sur les identités minoritaires : Black Studies, Women's Studies, Gay's Studies, Chicano Studies, etc. (ibid. : 15). L'affirmation de l'individu dans la pensée moderne puis postmoderne a elle aussi contribué à la popularité du terme. Cependant, le sens du concept ne semble pas encore fixé en sciences humaines (Mucchielli, [1986] $2011: 4)$.

En didactique des langues, l'identité est définie par Groux et Porcher (2003: 129) comme 'l'unité de l'individu ou un ensemble de traits permanents qu'il conserverait tout au long de sa vie'. Les auteurs précisent qu'il y a rarement concordance entre l'identité présentée par le sujet et celle attribuée par l'entourage. Ainsi, l'altérité se représente notre identité selon ses propres critères de catégorisation. Cependant, envisager l'identité comme un ensemble de traits permanents semble réducteur. Au contraire, le caractère évolutif et non figé des composantes de l'identité constitue selon nous l'essence de la notion. Comme le souligne Mucchielli ([1986] 2011 : 10), l'identité est en perpétuelle transformation et constitue un système complexe de significations :

L'identité est un ensemble de significations (variables selon les acteurs d'une situation) apposées par des acteurs sur une réalité physique et subjective, plus ou moins floue, de leurs mondes vécus, ensemble construit par un autre acteur. C'est donc un sens perçu donné par chaque acteur au sujet de lui-même ou d'autres acteurs.

Par ailleurs, l'identité s'actualise constamment dans l'interaction puisque l'individu se comporte dans celle-ci de façon à se sentir valorisé et à, par exemple, ne pas 'perdre la face', comme le montrent les travaux de Goffman (1973a, 1973b, 1974). L'individu peut choisir de mettre en adéquation la projection de son identité avec ce que l'altérité attend de lui et/ou modifier cette projection en fonction d'expériences singulières. 


\section{LES REPRÉSENTATIONS DE L’ALTÉRITÉ}

La représentation est un concept transversal issu de la sociologie (Durkheim, [1898] 1951) et de la psychologie (Piaget, 1936). Son étude en psychologie sociale a été reprise dans les années 1960 par Moscovici qui décide de spécifier les 'représentations sociales' en regard des 'représentations collectives' (Durkheim) et des 'représentations individuelles' (Piaget). Moscovici préfère parler de représentations sociales, Boyer de représentations communautaires tandis que d'autres didacticiens ont préféré les représentations collectives de Durkheim. Boyer (2003: 14) établit d'ailleurs une distinction entre les représentations sociales 'différenciatrices des groupes constitutifs d'une société selon les positions qu'ils occupent' et les représentations collectives 'consensuelles sur le plan de la communauté dans son ensemble'. Les premières seraient donc intra-communautaires et les secondes uniquement communautaires. Quand il traite des deux, l'auteur les réunit sous l'appellation 'représentations partagées', plus générale.

Pour Moscovici (1989: 82), les représentations sont dites sociales car elles se construisent justement dans les interactions sociales. Par ailleurs, elles sont évolutives et 'fournissent une grille de décodage, d'interprétation du monde et une matrice de sens qui jouent comme processus d'arbitrage de la réalité.' (Mannoni, [1998] 2010 : 91). Ce qui nous intéresse plus particulièrement dans l'analyse des manuels de langues, c'est justement le figement des identités et altérités induit par ces supports qui, d'une certaine façon, capturent l'ensemble des représentations circulant dans une société sur une autre. Ces ouvrages pédagogiques donnent à voir selon Zarate $(2006$ : 37) une 'simplification de la réalité sociale $[. .$.$] par la valorisation du recours à l'essentiel'.$

Les représentations sociales sont aujourd'hui définies en psychologie comme 'des ensembles complexes d'éléments, informations ou connaissances, partagés par un ensemble spécifique d'individus, à propos d'un objet donné' (Sales-Wuillemin, 2005: 179). Elles circulent dans cet ensemble spécifique d'individus et peuvent donc être parfois partagées et/ou diffusées par un groupe source sur un groupe cible. Dans une perspective didactique, Zarate (2006: 29) précise d'ailleurs que 'les représentations sociales constituent un mode à part entière de connaissance de la réalité, $[. .$.$] elles contribuent à faire exister une$ réalité dont elles sont constitutives'. Ainsi, toute représentation individuelle ou collective de l'altérité permet d'approcher une réalité, de la définir selon des filtres sans jamais pour autant prétendre à l'exactitude ni au réel.

Dans une perspective anthropologique, Augé (2010: 15-16) affirme que: 'Nous avons besoin d'autres qui "résistent", d'autres vraiment "autres", pour construire et développer notre identité ; nous avons besoin d'identifier les frontières de l'autre pour identifier les nôtres'.

Dès lors, de quelle façon réaffirme-t-on/définit-on ses propres frontières, sa propre identité collective ou, en tout cas, celle qui est perçue par l'altérité ? Nous le verrons en analysant de manière qualitative les contenus et discours de notre corpus. 


\section{L'IDENTITÉ COLLECTIVE DANS LES MANUELS FINLANDAIS}

\subsection{DESCRIPTION DU CORPUS}

Notre corpus est composé de deux séries de manuels de FLE utilisées en Finlande au collège et au lycée ; le premier dans les années $70: D$ 'accord, d'accord / Toujours d'accord et le second dans les années 2000 : Voilà. Comme Auger (2011 : 314), nous partons du postulat que l'énonciateur se repère en même temps qu'il repère l'Autre. En représentant l'Autre, ici le Français, l'énonciateur expose en même temps en quoi il est différent de lui. Il révèle son identité à travers l'énonciation, comme le fait tout orateur d'après Barthes ([1970] 1994 : 315) : 'L'orateur énonce une information et en même temps il dit : je suis ceci, je ne suis pas cela'. A travers la mise en discours de l'altérité, l'auteur-énonciateur diffuse à la fois des représentations sur l'autre, l'étranger, mais aussi sur sa communauté d'appartenance.

En outre, Auger (2003: 40) affirme que 'les procédés de valorisation/ dévalorisation sont constitutifs de toute communauté qui produit un discours sur un autre groupe'. Grâce à l'analyse de ces procédés, nous pouvons tenter de déceler l'image des sociétés source et cible véhiculée par les outils pédagogiques. Nous ferons référence aux extraits des manuels en utilisant les initiales de la série : DD pour D'accord, d'accord, TD pour Toujours d'accord, V suivi du numéro du volume pour Voilà (p.ex. V1 désigne Voilà, volume 1). Nous utilisons les caractères italiques pour mettre en relief les énoncés analysés.

\subsection{DE LA DÉPRÉCIATION DE L'IDENTITÉ COLLECTIVE SOURCE...}

Dans la série D'accord ? D'accord / Toujours d'accord, publiée dans les années 70, les références toponymiques permettent une comparaison chiffrée entre la France et la Finlande :

La France n'est qu'un point sur la carte du monde, mais c'est un point important. Sa position est centrale en Europe (tandis que la Finlande est située à la périphérie). La France est plus grande que la Finlande. Avec $550000 \mathrm{~km}^{2}$, la France est le plus grand pays d'Europe occidentale. (TD : 12)

La France a plus de 50 millions d'habitants. La population de la Finlande est beaucoup plus petite: moins de 5 millions d'habitants. La densité de la population est de 92 habitants au $\mathrm{km}^{2}$, c'est-à-dire bien inférieure à la densité des Pays-Bas (379), mais supérieure à la densité de la Suède (18) ou de la Finlande (14). (TD : 13)

La Finlande souffre de la comparaison avec le pays cible quant à sa position en Europe, sa superficie, la taille de sa population et sa densité. Le comparatif est même expliqué à l'aide de l'exemple suivant :

Le climat français est meilleur que celui de la Finlande. (TD : 22) 
Le comparatif de supériorité est lui aussi en faveur de la société cible: la France. Ces énoncés relatifs à la Finlande ne sont pas essentiels à la compréhension du point grammatical. Ce sont des choix auctoriaux et éditoriaux qui dévalorisent le pays source en valorisant l'altérité. La plupart des références à l'identité collective source, la Finlande, sont donc dévalorisantes et, en contrepoint, valorisent l'altérité française.

La Finlande est également perdante dans la confrontation à d'autres nations, p.ex. dans cet extrait du journal radio suisse qui annonce :

Championnat d'Europe : tour préliminaire : à Helsinki, les Pays-Bas ont battu la Finlande par 3 à 1 grâce à deux buts de Cruyff et un de Neskens. (TD : 164)

On assiste cependant à quelques tentatives de justification ou de déconstruction des représentations de l'autre sur soi, comme dans le chapitre 29 qui s'intitule 'La Finlande en raccourci' avec, par exemple, un texte émanant de l'altérité. Il s'agit d'un extrait du journal Sud-ouest dont l'auteur est français : Claude Rousseau.

L'énonciateur commence par définir l'identité finlandaise par la négative, c'est-à-dire par ce qu'elle n'est pas :

La Finlande ? Elle est dans une forme de vie, qui est d'ici, et de nulle part ailleurs. Qui n'est ni slave, $n i$ scandinave, $n i$ américaine.

Puis, par une gradation de l'amour pour la nature, qui semble être l'une de ses caractéristiques essentielles :

Elle est dans certains plats typiques venus tout droit de Carélie. Elle est, surtout, dans un amour total, exclusif, inimaginable pour la nature. Cela tient, je crois, à l'origine paysanne des Finlandais. Dès quarrivent les beaux jours, ils se ruent vers la nature, ils vont se cacher au fond des forêts, souvent dans une simple cabane, auprès d'un lac. Une cabane, à côté de laquelle il y en a toujours une autre, plus petite où fume la cheminée du sauna.

Dans la suite du texte, une polyphonie se met en place : l'auteur-énonciateur du manuel se décharge de la description de sa propre communauté et la confie à un énonciateur-relais (terme emprunté à Auger, 2007: 78), membre de la culture cible, l'auteur de l'article : Claude Rousseau. Celui-ci à son tour énonce les représentations que d'autres (ici, les gens du sud) peuvent avoir sur les Finlandais :

L'origine paysanne explique peut-être aussi les visages qui n'expriment rien, la retenue, qui semble si inhumaine à ceux qui viennent du Sud. Mais ce n'est qu'un masque. On ne montre pas ses sentiments dans la rue. On ne s'y embrasse pas. On ne se retourne pas sur un ivrogne couché entre deux voitures, sur un parking (des ivrognes, hélas, il y en a beaucoup). Mais c'est aussi, chez lui ou avec des amis, un homme très gai que ce 
Finlandais si réservé, si 'absent'. Qui rit très bien, qui plaisante, qui a le sens de l'humour. Chez les Finlandais, il y a sous la surface, un volcan! Mais c'est aussi le peuple le plus amical, le plus 'voisinant'. (TD : 128)

L'énonciateur initial et principal entend, par ce choix, briser les représentations circulant sur l'identité finlandaise en les expliquant par une origine ('paysanne'), explication dont la certitude est modalisée ('peut-être'). Les qualificatifs qui apparaissent comme des défauts d'après l'altérité ('absent', 'réservé') sont justifiés et même enrayés par des qualités et des superlatifs ('très gai', 'qui a le sens de l'humour', 'le plus amical', 'le plus "voisinant”'). Ce type de texte permet de douter du public cible : il semble qu'il s'adresse davantage à des apprenants de finnois qu'à des apprenants finlandais de français langue étrangère. Il me semble que l'auteur fournit ici à l'apprenant des arguments 'prêts à opposer' à toute représentation émise par l'altérité lors d'une interaction.

Dans cette série des années 1970, la subjectivité de l'énonciateur n'est pas très visible : il s'en remet souvent à d'autres discours mais, en effectuant les choix des documents, il fait indirectement entendre sa propre voix, à travers celles qu'il cautionne.

\section{3 ... À SA REVALORISATION}

L'axiologie des représentations, au départ défavorable à la Finlande, commence donc dans les années 70 à s'inverser, d'abord avec ces processus de déconstruction des auto-représentations puis à travers une valorisation de l'identité collective finlandaise, très nette dans les années 2000. En effet, dans la série parue trente ans plus tard intitulée Voilà, l'identité finlandaise est hautement valorisée, comme dans cet exemple où un journaliste québécois se rend dans un lycée finlandais :

Il veut en savoir un peu plus sur ce pays classé en tête des palmarès mondiaux des performances scolaires [...]. Vous savez sans doute que les résultats scolaires sont excellents et que la Finlande se situe dans le peloton de tête des pays de l'OCDE. [...] Il faut quand même signaler que les bases qu'on nous enseigne au collège sont solides. (V5:6-7)

Les auteurs insistent ici sur les performances éducatives mais montrent également, à travers le choix des personnages, que ces résultats intéressent les journalistes du monde entier. Le petit pays déprécié dans les années 70 devient un modèle à suivre dans différents domaines :

- La politique familiale

Un congé parental est normal en Finlande. (V4 : 50)

- Le droit des femmes

La Finlande fait néanmoins figure de pionnière, car elle est le premier pays $d u$ monde à avoir accordé aux femmes le droit de se faire élire. (V6: 128)

- La propreté, la salubrité, etc. 
En Finlande, on n'achète pas d'eau en bouteille parce que l'eau du robinet est bonne. (V4:12)

Le fait d'utiliser dans les exemples 1 et 3 le toponyme 'en Finlande' oppose la culture source et la culture cible et renvoie implicitement à l'anormalité des autres fonctionnements : le congé parental n'est pas un acquis social normal pour l'Autre et les Français boivent de l'eau en bouteille car la qualité de l'eau du robinet dans leur pays laisse à désirer. Dans l'exemple 2, l'auteur souhaite montrer que sa société d'appartenance se distingue également à l'échelle internationale. La seule occurrence dévalorisante sur la Finlande concerne la taille du pays, sur laquelle l'identité source n'a aucune prise :

Les Français lisent beaucoup de BD, mais la Finlande est un petit pays et les éditeurs ne s'y intéressent pas vraiment. (V2:139)

La dépréciation porte donc sur un paramètre que les Finlandais ne pourront pas changer. Toutes les autres références sont d'une axiologie positive.

Les coutumes finlandaises ne sont pas seulement décrites du point de vue source mais aussi à travers le regard que les personnages français portent sur elles :

Lauri : Nous, en Finlande, on se baigne même en hiver quand la mer est gelée. On fait un trou dans la glace et après le sauna, on se jette dans l'eau glacée.

Alexandra et Yves : Ils sont fous ces Finlandais! (V2: 203)

Non mais ça va pas la tête ! Tu veux notre mort ! [...] nous n'en croyions pas nos oreilles. Nous baigner, par un froid pareil ? [...] Curieusement, la deuxième fois, il était déjà bien plus facile de supporter la chaleur et peu à peu, nous avons commencé à apprécier ces coutumes finlandaises, y compris la baignade dans l'eau glacée. (V3 : 120)

Les personnages chargés de représenter l'altérité française (Alexandra et Yves) apprécient peu à peu les coutumes qui les choquaient au préalable. En réalité, l'énonciateur met ici en discours les étapes successives qui permettent le développement de l'ethnorelativisme chez l'apprenant, en montrant que les personnages sont aussi passés par ces étapes avant de comprendre et d'accepter l'altérité. Il s'agit en quelque sorte d'une mise en abyme de l'approche interculturelle prônée par les directives nationales et même supra-nationales comme le Conseil de l'Europe.

\section{CONCLUSION}

Pour conclure, l'identité est une notion complexe car mouvante, faite d'appartenances multiples qui s'opposent parfois entre elles: identité régionale, nationale, européenne, occidentale, religieuse, sexuelle, professionnelle, etc. De plus, l'identité est évolutive: elle change avec le temps et modifie 
les comportements selon les âges, les contextes, les circonstances. Il est par conséquent difficile de la capturer et de la décrire. C'est la raison pour laquelle nous avons choisi de travailler sur les discours sur l'identité et l'altérité, qui cristallisent et produisent des représentations dans un support figé comme le manuel scolaire, car celui-ci donne à voir la projection d'une identité collective par l'auteur-énonciateur, ainsi que sa perception par l'altérité à une époque donnée. De la même façon, cette étude permet de mettre au jour l'orientation axiologique de l'énonciateur envers l'identité (Buchart, 2018), ici dévalorisante dans les années 70 puis promotionnelle et laudative dans les années 2000.

L'individu définit en général son identité individuelle ou collective en contrepoint par rapport à celle des autres, en affirmant ce qu'il n'est pas et en se définissant comme faisant partie d'un groupe partageant cette identité-là (p.ex. catholique vs. musulman, hétérosexuel vs. homosexuel, Européen vs. Africain, cadre vs. ouvrier, etc.). Comme l'explique Mucchielli ([1986] 2011: 3) : 'les fondements de l'identité d'un acteur pour d'autres acteurs (ou pour lui-même) se trouvent dans les identités de ces autres acteurs (ou du premier acteur lui-même)'. L'individu a donc besoin de classifications, de catégorisations et de structures de référence pour se situer dans un système, ce que propose le manuel de langue malgré tous les reproches qu'on peut lui faire.

\section{BIBLIOGRAPHIE}

Augé, M. (2010) La Communauté illusoire. Paris : Petite bibliothèque.

Auger, N. (2003) Les représentations interculturelles dans des manuels de français langue étrangère en usage dans l'Union Européenne. In C. Alén-Garabato, N. Auger, P. Gardies et E. Kotul (éds.) Les représentations interculturelles en didactique des langues-cultures: enquêtes et analyses (pp. 35-71). Paris : L'Harmattan.

Auger, N. (2007) Constructions de l'interculturel dans les manuels de langue. Cortil-Wodon (Belgique) : Editions Modulaires Européennes (E.M.E.), coll. Proximités : didactique.

Auger, N. (2011) Les manuels : analyser les discours. In P. Blanchet et P. Chardenet (dirs.) Guide pour la recherche en didactique des langues et culture : approches contextualisées (pp. 313-316). Paris : Editions des Archives contemporaines.

Barthes, R. ([1970] 1994) Lancienne rhétorique. Aide-mémoire. In Recherches rhétoriques (pp. 172-223). Paris : Points (1e éd. Communications 16).

Boyer, H. (2003) De l'autre côté du discours, recherches sur le fonctionnement des représentations communautaires. Paris : L'Harmattan.

Buchart, M. (2018) La matérialité discursive des représentations de l'altérité française dans les manuels de FLE finlandais : axiologie de la description, subjectivèmes et généralisations. In S. Dubois, J. Montemayor Gracia et V. Neusius (éds.) Lehrwerke für Sprache und Literatur als kulturelle Mittler im Fremdsprachenunterricht: Québec - Kanada - Europa / Les manuels de langue et de littérature étrangères comme médiateurs culturels: Québec-Canada - Europe (pp. 65-79). St. Ingbert: Röhrig Universitätsverlag.

Durkheim, E. (1898 [1951]) Sociologie et philosophie. Paris : PUF. Réédition de Durkheim E. Représentations individuelles et représentations collectives. In Revue de Métaphysique et de Morale, VI. (pp. 273-302).

Goffman, E. (1973a) La mise en scène de la vie quotidienne. Paris : Editions de Minuit. 
Goffman, E. (1973b) La mise en scène de la vie quotidienne : 2. les relations en public. Paris : Editions de Minuit.

Goffman, E. (1974) Les rites d'interaction. Paris : Editions de Minuit.

Groux, D. et Porcher, L. (2003) L'altérité. Paris : L'Harmattan.

Halpern, C. (2004) Faut-il en finir avec l'identité ? In C. Halpern et J-C. Ruano-Borbalan (coord.) Identité(s). L'individu. Le groupe. La société (pp. 11-20). Auxerre : Sciences humaines éditions.

Mannoni, P. ([1998] 2010) Les représentations sociales. Paris : PUF.

Moscovici, S. (1989) Des représentations collectives aux représentations sociales : éléments pour une histoire. In D. Jodelet (éd.) Les représentations sociales (pp. 79-103). Paris : PUF.

Mucchielli, A. ([1986] 2011) L'identité. Paris : PUF.

Piaget, J. (1936) La naissance de l'intelligence chez l'enfant. Neuchâtel, Paris : Delachaux et Nieslé.

Porcher, L. (1997) Lever de rideau. In G. Zarate and M. Candelier (coord.), Les représentations en Didactique des Langues et des Cultures, (pp. 11-27). Paris : ENS de Fontenay/St Cloud.

Sales-Wuillemin, E. (2005) Psychologie sociale expérimentale de l'usage du langage : représentations sociales, catégorisation et attitudes : perspectives nouvelles. Paris : L'Harmattan.

Zarate, G. ([1993] 2006) Représentation de l'étranger et didactique des langues. Paris : Didier.

\title{
CORPUS DE MANUELS
}

Anttila, J., Nuotio, I. et Sinisalo, M. ([1977] 1980) D’accord ? D’accord. Jyväskylä : K. G. Gummerus Osakeyhtiönkirjapaino.

Anttila, J., Nuotio, I. et Sinisalo, M. (1977) Toujours d'accord. Helsinki : Yhteiskirjapaino Oy.

Bärlund, K., Jokinen, J., Raitala, E., Bellotti, L. et Laspeyres, C. ([2004] 2008) Voilà ! 1 Textes. Keuruu : Otavan Kirjapaino Oy.

Bärlund, K., Jokinen, J., Maala, S., Mauffret, D. et Raitala, E. (2009) Voilà ! 2 Textes et exercices. Les loisirs. Keuruu : Otavan Kirjapaino Oy.

Bärlund, K., Raitala, E., Simula, P. et Mauffret, D. ([2005] 2008) Voilà! 3 Textes et exercices. Chez nous et ailleurs. Keuruu : Otavan Kirjapaino Oy.

Hankala-Perttula, P., Mauffret, D., Raitala, E. et Simula, P.( [2006] 2009) Voilà ! 4 Textes et exercices. Avant et maintenant. Keuruu : Otavan Kirjapaino Oy.

Hankala-Perttula, P., Kivivirta, N., Kuikka, T., Mauffret, D. et Raitala, E. ([2007] 2009) Voilà ! 5 Textes et exercices. Les études et les projets d'avenir. Keuruu : Otavan Kirjapaino Oy.

Kivivirta, N., Kuikka, T., Mauffret, D. et Raitala, E. ([2007] 2008) Voilà ! 6 Textes et exercices. La culture; Notre monde à nous tous. Keuruu : Otavan Kirjapaino Oy.

\section{PROJECTION VS PERCEPTION: PRESENTING OF THE SELF THROUGH THE DESCRIPTION OF THE OTHER IN DIDACTICS OF FFL}

\begin{abstract}
In this paper, we aim at problematizing the notions of identity/the self and alterity/the other, from the perspective of discourse analysis and didactics of interculturality in teaching and learning FFL (French as a Foreign Language). After explaining what different fields have brought to the definition of these notions, we will particularly focus on the representations of collective identity in language courses. Indeed, through the discourse on alterity, the enunciator (for instance, the author of an FFL textbook) spreads frozen representations of the otherness, the 'foreigner', but also of the self, the 'source culture' he
\end{abstract}


belongs to. Therefore, through local textbook discourses, the researcher can detect and reveal what the source society questions, values or depreciates, finds interesting, weird or abnormal in the target society. The produced discourse mirrors two worlds meeting by means of a constant game of endogenous and exogenous representations. We will analyze diachronically some examples of this axiological way of presenting oneself in pedagogical material in Finland and show how the axiology of self-representations has been reversed.

Key words: identity, otherness, textbooks, didactics, representations

Mélanie Buchart (Dr. en sciences du langage) est maître de conférences en philologie française à l'Université de Helsinki (Finlande). Ses intérêts de recherche portent sur la conceptualisation de la culture et de l'interculturel en didactique du FLE et plus généralement sur les discours sur l'identité et l'altérité (p.ex.le discours de haine en ligne).Courriel : melanie.buchart@helsinki.fi 